\title{
INFECÇÕES DA CORRENTE SANGUÍNEA ASSOCIADAS AO CATETER VENOSO CENTRAL EM UNIDADE DE TERAPIA INTENSIVA: REVISÃO INTEGRATIVA DA LITERATURA
}

\author{
BLOODSTREAM INFECTIONS ASSOCIATED WITH CENTRAL VENOUS \\ CATHETERS IN THE INTENSIVE CARE UNIT: INTEGRATIVE LITERATURE \\ REVIEW
}

\author{
Camila Monteiro Bueno ${ }^{1}$ \\ Mayara Emili Basilio dos Santos ${ }^{2}$ \\ Morgana Kivea de Azevedo 3 \\ Elaine Reda da Silva ${ }^{4}$
}

RESUMO: Este estudo teve como objetivo analisar a produção científica nacional relacionada com a prevenção das infecções da corrente sanguínea associada ao cateter venoso central (CVC) em Unidade de Terapia Intensiva. Realizou-se uma revisão integrativa através da base de dados Biblioteca Virtual em Saúde (BVS), especificamente, Literatura Latino Americana e do Caribe em Ciências da Saúde (LILACS), Medical Literature Analysis and Retrieval Sistem On-line (Medline) e Base de dados em Enfermagem (BDENF). Dos 9 artigos selecionados, 4 abordaram a adesão dos profissionais frente aos métodos de prevenção das infecções da corrente sanguínea associada ao CVC; 3 as intervenções para prevenção das infecções da corrente sanguínea associadas ao CVC e 20 conhecimento dos métodos de prevenção das infecções da corrente sanguínea associada ao CVC. Concluiu-se que se torna importante ações de educação permanente acerca das medidas de prevenção de infecção de corrente sanguínea associada ao uso de cateter venoso central.

Descritores: Infecções relacionadas a cateter. Unidade de Terapia Intensiva. Cuidados de Enfermagem.

ABSTRACT: This study aimed to analyze the national scientific content related to the prevention of bloodstream infections associated with central venous catheters (CVC) in the Intensive Care Unit. An integrative review was carried out using the Virtual Health

\footnotetext{
${ }^{1}$ Estudante de Graduação do Curso de Enfermagem da Universidade São Francisco - USF. Técnica de Enfermagem pela Escola Biotec Atibaia-SP. Atuou como estagiária na Empresa Vale Diagnósticos Laboratorial Atibaia/SP. E-mail: cadu270914@gmail.com

${ }^{2}$ Estudante de Graduação do Curso de Enfermagem da Universidade São Francisco - USF. Auxiliar de farmácia pela Escola RT Meta de Bragança Paulista. Atuou como estagiária na Prefeitura Municipal de Extrema/MG - Setor da Saúde. E-mail: mayara-basilioi@hotmail.com

${ }^{3}$ Estudante de Graduação do Curso de Enfermagem da Universidade São Francisco - USF. Atuou como estagiária na empresa Vale Diagnóstico Laboratorial Atibaia/SP. E-mail: morganakazevedo@gmail.com

4 Professora de Graduação na Área da Saúde da Universidade São Francisco - USF. Enfermeira pela Faculdade de Enfermagem do Hospital Israelita Albert Einstein. Mestre pelo Programa de Pós-Graduação na Saúde do Adulto pela Universidade de São Paulo - USP. Especialista em Enfermagem Cirúrgica pela Universidade Federal de São Paulo-UNIFESP. E-mail: reda.elaines@gmail.com
} 
Library (VHL) database, specifically, Latin American and Caribbean Literature on Health Sciences (LILACS), Medical Literature Analysis and Retrieval Online System (Medline) and Database data in Nursing (BDENF). Of the 9 articles selected, 4 addressed the adherence of professionals to methods of prevention of bloodstream infections associated with CVC; 3 interventions to prevent CVC-associated bloodstream infections and 2 knowledge of methods to prevent CVC-associated bloodstream infections. It was concluded that education actions about measures to prevent bloodstream infection associated with the use of central venous catheters becomes importante.

Descriptors: Catheter-related infections. Intensive Care Unit. Nursing Care.

\section{INTRODUÇÃO}

A infecção relacionada ao uso de cateter venoso central (CVC) ocupa o terceiro lugar de causas de infecções hospitalares (SILVEIRA et al, 20ı0). Esse tipo de infecção interfere diretamente na saúde do paciente, no tipo de tratamento, no aumento da sua permanência no ambiente hospitalar e, além disso, nos indicadores da qualidade da assistência prestada (MARQUES NETTO et al, 2009.)

Os cateteres venosos centrais (CVC) são acessos vasculares utilizados para infusão de medicações, soluções endovenosas, hemoderivados e quimioterápicos em pacientes com

limitação de acesso venoso periférico e, ainda, para nutrição parenteral prolongada, monitorização hemodinâmica invasiva da pressão sanguínea arterial, pressão venosa central, pressão da artéria pulmonar, medição de débito cardíaco e acesso para hemodiálise (SANTOS; LEAL; CAVALHEIRO, 2006; ROSADO; ROMANELLI; CAMARGOS, 20II).

A escolha do tipo de dispositivo varia de acordo com o tempo de permanência necessário, podendo ser classificado como cateter de curta ou longa permanência. Em casos de uso prolongado é indicado o CVC que possui mecanismos que evitam a colonização bacteriana via extra luminal, cuja instalação é cirúrgica (CDC, 2016; ANVISA, 2017).

De acordo com a Agência Nacional de Vigilância Sanitária (ANVISA) as Infecções Primárias de Corrente Sanguínea (IPCS) estão geralmente relacionadas à assistência à saúde e são infecções de consequências sistêmicas graves, na maioria das vezes sem foco primário identificável. Os fatores de risco mais comuns, são os cateteres vasculares centrais e principalmente os de curta permanência (ANVISA, 2017). 
As infecções da corrente sanguínea são multifatoriais e apresentam fisiopatologia, critérios diagnósticos, implicações terapêuticas, prognósticas e preventivas distintas (ANVISA, 2009).

Logo, verifica-se que os cateteres venosos centrais são essenciais na assistência à saúde, particularmente na Unidade de Terapia Intensiva (UTI), que tem por finalidade a assistência ao paciente crítico.

Por outro lado, sabe-se que em UTIs, os riscos de infecções podem aumentar, pois há uma ampla variedade de microrganismos que, muitas vezes, são multirresistentes e que ao entrar em contato com pacientes imunologicamente debilitados pode contribuir para agravar o quadro inicial.

Embora o CVC forneça acesso vascular seguro, as práticas inadequadas em seu manuseio podem acarretar maior risco de diversas complicações para os pacientes, incluindo a infecção de corrente sanguínea associada ao cateter venoso central (PIEROTTO, 2015).

O risco de infecção, relacionado ao acesso vascular, está associado à localização do acesso, solução infundida, experiência do profissional que realiza o procedimento, tempo de permanência, tipo e manipulação do cateter, entre outros (FERNANDES; RIBEIRO, 2000). Assim, tais fatores constituem pontos estratégicos importantes para ações preventivas dessas infecções.

Para a Agência Nacional de Vigilância Sanitária (ANVISA), a Infecção Primária de Corrente Sanguínea associada a Cateter Venoso Central (IPCS-CVC) corresponde à Infecção Relacionada à Assistência à Saúde (IRAS) com maior potencial preventivo, ou seja, principal tipo de infecção que pode ser evitável (ANVISA, 2017).

Desta forma, verifica-se que toda a equipe de saúde deve apresentar vigilância a todos os procedimentos que envolvem o acesso vascular, prevenindo e controlando possíveis complicações relacionadas a esse cateter. Porém, a equipe de enfermagem que está diretamente responsável pela assistência e está envolvida desde a implantação até o manuseio do CVC, pode atuar de forma mais eficaz na profilaxia e controle de infecções relacionadas ao uso desse dispositivo (MENDONÇA, et al, 2oir).

O enfermeiro possui papel essencial na prevenção da infecção de corrente sanguínea relacionada a cateteres centrais, pois a manipulação do dispositivo, 
principalmente a troca de curativo, é de responsabilidade deste profissional, sendo responsável também pela identificação e notificação dos casos de infecção associada aos cuidados em saúde e maior possibilidade de atuação na profilaxia e controle das IRAS ((MENDONÇA, et al, 2oII).

Considerando que os procedimentos de enfermagem podem desempenhar um papel importante na ocorrência de infecção, vale ressaltar não só a importância da utilização de protocolos específicos para a inserção do CVC, mas, também, durante a manipulação desse dispositivo, na administração de medicamentos, monitorização e troca de curativo. Além disso, verifica-se a importância da adoção de políticas que visem o aprimoramento dos profissionais afim de diminuir a incidência dessa infecção (CATARINO et al, 2012).

Com base no exposto, este estudo teve como objetivo analisar a produção científica nacional relacionada com a prevenção das infecções da corrente sanguínea associada ao cateter venoso central em Unidade de Terapia Intensiva.

\section{MATERIAL E MÉTODO}

Tratou-se de uma revisão integrativa relacionada à prevenção das infecções da corrente sanguínea associada ao cateter venoso central em Unidade de Terapia Intensiva.

Este tipo de estudo determina o conhecimento atual sobre uma temática específica, sintetizando resultados obtidos sobre um tema, de maneira sistemática, ordenada e abrangente (ERCOLE; MELO; ALCOFORATO, 2014).

Formulou-se a seguinte questão norteadora: Quais intervenções estão sendo realizadas visando a prevenção de infecções da corrente sanguínea associada ao cateter venoso central em Unidade de Terapia Intensiva?

O levantamento dos artigos, foi realizado através da busca de publicações indexadas na base de dados Biblioteca Virtual em Saúde (BVS), especificamente, Literatura Latino Americana e do Caribe em Ciências da Saúde (LILACS), Medical Literature Analysis and Retrieval Sistem On-line (Medline) e Base de dados em Enfermagem (BDENF), nos meses de fevereiro e março de 202I, através dos Descritores em Ciências da Saúde (DECS): Infecções relacionadas a cateter, Unidade de Terapia Intensiva, Cuidados de Enfermagem.

Foram estabelecidos os seguintes critérios de inclusão dos estudos para comporem a revisão: artigos publicados no período de 2017 a 2021; estar escrito em português; estar 
disponível eletronicamente na íntegra e abordar a temática intervenção de enfermagem relacionada ao cateter venoso central. Os critérios de exclusão foram: artigos repetidos, não disponíveis eletronicamente, não compatíveis com a temática abordada neste estudo e materiais bibliográficos como dissertações e teses.

Inicialmente foram identificados os estudos a partir da busca nas bases de dados, em seguida foi realizada a leitura preliminar de títulos e resumos e posteriormente a leitura dos artigos na íntegra, sendo que estes critérios, referentes a busca dos artigos, foram representados em forma de fluxograma conforme representado na figura I.

Para a organização dos dados, foi elaborado um quadro contendo: título, ano de publicação, autor, periódico, objetivo e área temática (Quadro I).

A análise dos dados foi realizada de forma descritiva a partir do agrupamento das áreas temáticas relacionadas ao material bibliográfico encontrado.

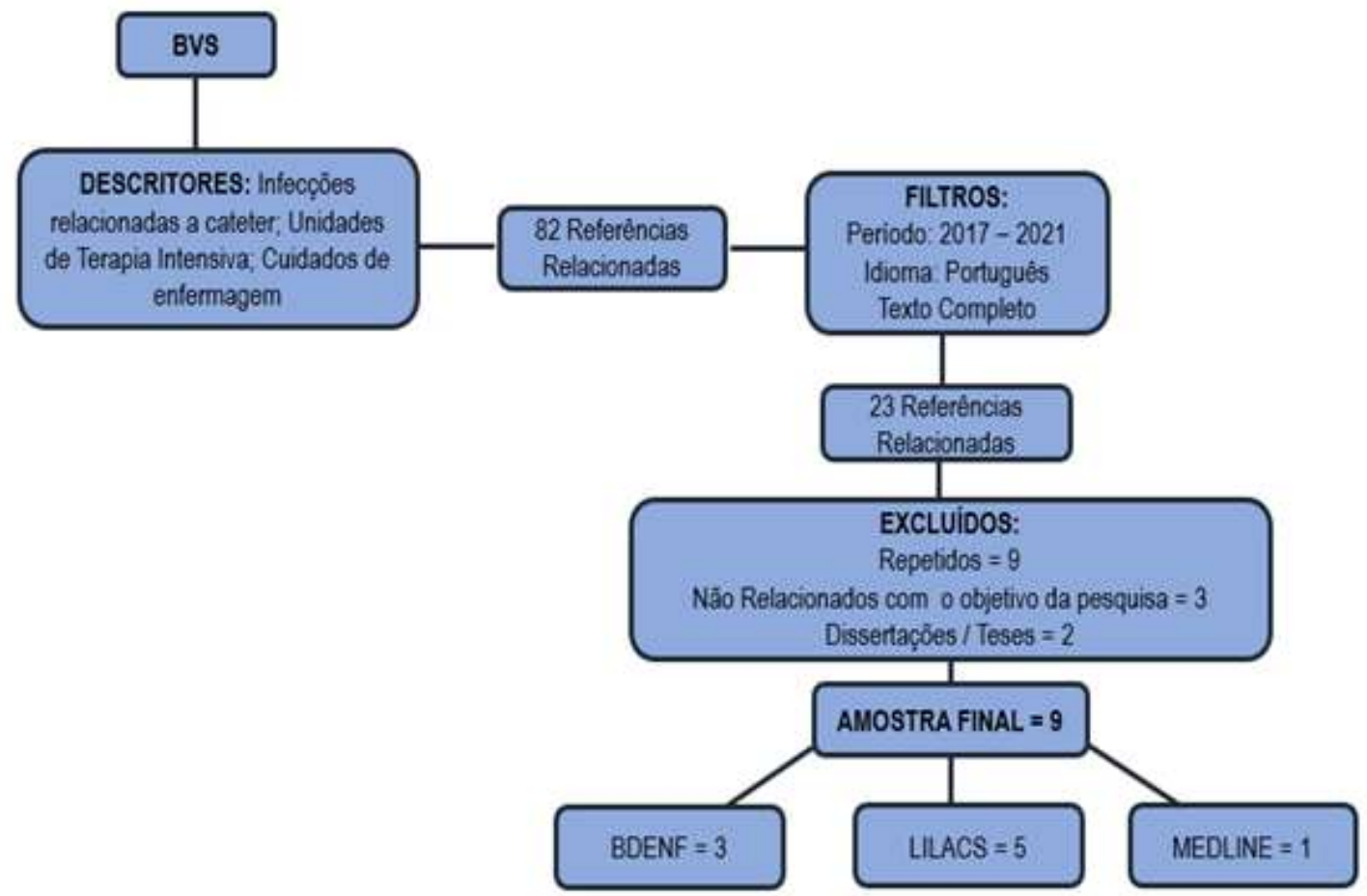

Figura 1 - Descrição da seleção dos artigos científicos, 2017-2021.

Fonte: próprias autoras.

\section{RESULTADOS E DISCUSSÃO}

A caracterização dos estudos quanto ao título, ano de publicação, autor, periódico, objetivo e área temática foram organizados em forma de quadro para facilitar a análise (Quadro I). 
Quadro I - Identificação dos artigos inseridos na revisão de literatura segundo título, ano de publicação, autor, periódico, objetivo e área temática, 2017-2021.

\begin{tabular}{|c|c|c|c|c|c|c|}
\hline $\begin{array}{l}\mathbf{N} \\
\underline{\mathbf{o}}\end{array}$ & $\begin{array}{l}\text { TÍTULO DO } \\
\text { ARTIGO }\end{array}$ & $\begin{array}{c}\text { AN } \\
\mathrm{O}\end{array}$ & AUTOR & $\begin{array}{c}\text { PERIÓDIC } \\
\text { O }\end{array}$ & OBJETIVO & $\begin{array}{c}\text { ÁREA } \\
\text { TEMÁTI } \\
\text { CA }\end{array}$ \\
\hline I & $\begin{array}{c}\text { Medidas } \\
\text { utilizadas em } \\
\text { unidades de } \\
\text { terapia } \\
\text { intensiva para } \\
\text { prevenção de } \\
\text { infecção: } \\
\text { revisão } \\
\text { integrativa }\end{array}$ & 2020 & $\begin{array}{c}\text { Dantas et } \\
\text { al }\end{array}$ & Rev Rene & $\begin{array}{c}\text { Verificar } \\
\text { medidas } \\
\text { utilizadas em } \\
\text { unidades de } \\
\text { terapia } \\
\text { intensiva para } \\
\text { prevenção de } \\
\text { infecção } \\
\text { relacionada à } \\
\text { assistência à } \\
\text { saúde }\end{array}$ & $\begin{array}{c}\text { Intervençõ } \\
\text { es para } \\
\text { prevenção } \\
\text { das } \\
\text { infecções } \\
\text { da corrente } \\
\text { sanguínea } \\
\text { associada } \\
\text { ao cateter } \\
\text { venoso } \\
\text { central }\end{array}$ \\
\hline 2 & $\begin{array}{c}\text { Bundle de } \\
\text { Cateter } \\
\text { Venoso } \\
\text { Central: } \\
\text { conhecimento } \\
\text { e } \\
\text { comportament } \\
\text { o de } \\
\text { profissionais } \\
\text { em Unidades } \\
\text { de Terapia } \\
\text { Intensiva } \\
\text { adulto }\end{array}$ & 2020 & $\begin{array}{c}\text { Costa et } \\
\text { al }\end{array}$ & $\begin{array}{l}\text { Rev. esc. } \\
\text { enferm. USP }\end{array}$ & $\begin{array}{c}\text { Avaliar } \\
\text { conhecimento e } \\
\text { comportamento } \\
\text { dos } \\
\text { profissionais de } \\
\text { Unidades de } \\
\text { Terapia } \\
\text { Intensiva } \\
\text { quanto às ações } \\
\text { recomendadas } \\
\text { no bundle de } \\
\text { prevenção de } \\
\text { infecção de } \\
\text { corrente } \\
\text { sanguínea } \\
\text { relacionada ao } \\
\text { cateter venoso } \\
\text { central. }\end{array}$ & $\begin{array}{l}\text { Conhecime } \\
\text { nto dos } \\
\text { métodos de } \\
\text { prevenção } \\
\text { das } \\
\text { infecções } \\
\text { da corrente } \\
\text { sanguínea } \\
\text { associada } \\
\text { ao cateter } \\
\text { venoso } \\
\text { central }\end{array}$ \\
\hline 3 & $\begin{array}{l}\text { Bundle para a } \\
\text { prevenção de } \\
\text { infecção de } \\
\text { corrente } \\
\text { sanguínea }\end{array}$ & 2019 & $\begin{array}{l}\text { Fernande } \\
\text { s et al. }\end{array}$ & $\begin{array}{c}\text { Rev enferm } \\
\text { UFPE }\end{array}$ & $\begin{array}{c}\text { Verificar o } \\
\text { conhecimento } \\
\text { dos } \\
\text { profissionais } \\
\text { intensivistas } \\
\text { sobre o bundle } \\
\text { para a } \\
\text { prevenção de } \\
\text { infecção de } \\
\text { corrente } \\
\text { sanguínea }\end{array}$ & $\begin{array}{l}\text { Intervençõ } \\
\text { es para } \\
\text { prevenção } \\
\text { das } \\
\text { infecções } \\
\text { da corrente } \\
\text { sanguínea } \\
\text { associada } \\
\text { ao cateter } \\
\text { venoso } \\
\text { central }\end{array}$ \\
\hline
\end{tabular}




\begin{tabular}{|c|c|c|c|c|c|c|}
\hline & & & & & $\begin{array}{c}\text { relacionada ao } \\
\text { cateter venoso } \\
\text { central e sobre } \\
\text { os cuidados no } \\
\text { manejo deste } \\
\text { dispositivo. }\end{array}$ & \\
\hline 4 & $\begin{array}{l}\text { Adesão da } \\
\text { enfermagem } \\
\text { ao protocolo de } \\
\text { infecção de } \\
\text { corrente } \\
\text { sanguínea }\end{array}$ & 2018 & $\begin{array}{l}\text { Crivelaro } \\
\text { et al. }\end{array}$ & $\begin{array}{c}\text { Rev enferm } \\
\text { UFPE }\end{array}$ & $\begin{array}{c}\text { Verificar a } \\
\text { adesão da } \\
\text { equipe de } \\
\text { Enfermagem ao } \\
\text { protocolo de } \\
\text { infecção de } \\
\text { corrente } \\
\text { sanguínea em } \\
\text { pacientes em } \\
\text { uso de cateteres } \\
\text { intravasculares }\end{array}$ & $\begin{array}{c}\text { Adesão dos } \\
\text { profissiona } \\
\text { is frente } \\
\text { aos } \\
\text { métodos de } \\
\text { prevenção } \\
\text { das } \\
\text { infecções } \\
\text { da corrente } \\
\text { sanguínea } \\
\text { associada } \\
\text { ao cateter } \\
\text { venoso } \\
\text { central }\end{array}$ \\
\hline 5 & $\begin{array}{l}\text { Conhecimento } \\
\text { autorreferido } \\
\text { das equipes } \\
\text { médica e de } \\
\text { enfermagem } \\
\text { quanto às } \\
\text { medidas de } \\
\text { prevenção de } \\
\text { infecção da } \\
\text { corrente } \\
\text { sanguínea }\end{array}$ & 2018 & $\begin{array}{c}\text { Silva; } \\
\text { Oliveira }\end{array}$ & $\begin{array}{c}\text { Texto } \\
\text { contexto - } \\
\text { enferm }\end{array}$ & $\begin{array}{c}\text { Avaliar o } \\
\text { conhecimento } \\
\text { autorreferido } \\
\text { das equipes } \\
\text { médica e de } \\
\text { enfermagem } \\
\text { quanto às } \\
\text { medidas de } \\
\text { prevenção de } \\
\text { infecção da } \\
\text { corrente } \\
\text { sanguínea } \\
\text { relacionada ao } \\
\text { cateter venoso } \\
\text { central. }\end{array}$ & $\begin{array}{l}\text { Conhecime } \\
\text { nto dos } \\
\text { métodos de } \\
\text { prevenção } \\
\text { das } \\
\text { infecções } \\
\text { da corrente } \\
\text { sanguínea } \\
\text { associada } \\
\text { ao cateter } \\
\text { venoso } \\
\text { central }\end{array}$ \\
\hline 6 & $\begin{array}{l}\text { Positive } \\
\text { Deviance } \\
\text { como } \\
\text { estratégia na } \\
\text { prevenção e } \\
\text { controle das } \\
\text { infecções de } \\
\text { corrente } \\
\text { sanguínea na } \\
\text { terapia } \\
\text { intensiva }\end{array}$ & 2017 & $\begin{array}{l}\text { Oliveira } \\
\text { et al. }\end{array}$ & $\begin{array}{c}\text { Rev Esc } \\
\text { Enferm USP }\end{array}$ & $\begin{array}{c}\text { Descrever a } \\
\text { aplicação do } \\
\text { Positive } \\
\text { Deviance como } \\
\text { estratégia na } \\
\text { prevenção e no } \\
\text { controle da } \\
\text { infecção de } \\
\text { corrente } \\
\text { sanguínea. }\end{array}$ & $\begin{array}{c}\text { Intervençõ } \\
\text { es para } \\
\text { prevenção } \\
\text { das } \\
\text { infecções } \\
\text { da corrente } \\
\text { sanguínea } \\
\text { associada } \\
\text { ao cateter } \\
\text { venoso } \\
\text { central }\end{array}$ \\
\hline
\end{tabular}




\begin{tabular}{|c|c|c|c|c|c|c|}
\hline & & & & & & \\
\hline 7 & $\begin{array}{l}\text { Adesão às } \\
\text { medidas para } \\
\text { prevenção da } \\
\text { infecção da } \\
\text { corrente } \\
\text { sanguínea } \\
\text { relacionada ao } \\
\text { cateter venoso } \\
\text { central }\end{array}$ & 2017 & $\begin{array}{c}\text { Silva; } \\
\text { Oliveira }\end{array}$ & $\begin{array}{l}\text { Enferm. } \\
\text { Foco }\end{array}$ & $\begin{array}{l}\text { Verificar a } \\
\text { adesão da } \\
\text { equipe } \\
\text { multiprofission } \\
\text { al para as } \\
\text { medidas de } \\
\text { prevenção da } \\
\text { infecção da } \\
\text { corrente } \\
\text { sanguínea } \\
\text { relacionada ao } \\
\text { cateter venoso } \\
\text { central. }\end{array}$ & $\begin{array}{l}\text { Adesão dos } \\
\text { profissiona } \\
\text { is frente } \\
\text { aos } \\
\text { métodos de } \\
\text { prevenção } \\
\text { das } \\
\text { infecções } \\
\text { da corrente } \\
\text { sanguínea } \\
\text { associada } \\
\text { ao cateter } \\
\text { venoso } \\
\text { central }\end{array}$ \\
\hline 8 & $\begin{array}{c}\text { Adesão ao } \\
\text { bundle de } \\
\text { inserção de } \\
\text { cateter venoso } \\
\text { central em } \\
\text { unidades } \\
\text { neonatais e } \\
\text { pediátricas }\end{array}$ & 2017 & $\begin{array}{c}\text { Araújo et } \\
\text { al }\end{array}$ & $\begin{array}{c}\text { Rev Esc } \\
\text { Enferm USP }\end{array}$ & $\begin{array}{l}\text { Descrever o } \\
\text { comportamento } \\
\text { observado dos } \\
\text { profissionais da } \\
\text { Unidade de } \\
\text { Terapia } \\
\text { Intensiva } \\
\text { Neonatale } \\
\text { Pediátrica } \\
\text { segundo os itens } \\
\text { do bundle de } \\
\text { inserção de } \\
\text { cateter venoso } \\
\text { central, bem } \\
\text { como o perfil } \\
\text { clínico e de } \\
\text { nascimento de } \\
\text { neonatos e } \\
\text { crianças que } \\
\text { receberam os } \\
\text { dispositivos. }\end{array}$ & $\begin{array}{c}\text { Adesão dos } \\
\text { profissiona } \\
\text { is frente } \\
\text { aos } \\
\text { métodos de } \\
\text { prevenção } \\
\text { das } \\
\text { infecções } \\
\text { da corrente } \\
\text { sanguínea } \\
\text { associada } \\
\text { ao cateter } \\
\text { venoso } \\
\text { central }\end{array}$ \\
\hline 9 & $\begin{array}{c}\text { Adesão da } \\
\text { equipe de } \\
\text { enfermagem às } \\
\text { medidas de } \\
\text { prevenção de } \\
\text { infecções de } \\
\text { corrente } \\
\text { sanguínea }\end{array}$ & 2017 & $\begin{array}{c}\text { Dantas et } \\
\text { al }\end{array}$ & $\begin{array}{c}\text { Rev enferm } \\
\text { UFPE }\end{array}$ & $\begin{array}{c}\text { Avaliar o } \\
\text { conhecimento e } \\
\text { adesão da } \\
\text { equipe de } \\
\text { enfermagem às } \\
\text { medidas de } \\
\text { prevenção de } \\
\text { infecções de } \\
\text { corrente } \\
\text { sanguínea }\end{array}$ & $\begin{array}{l}\text { Adesão dos } \\
\text { profissiona } \\
\text { is frente } \\
\text { aos } \\
\text { métodos de } \\
\text { prevenção } \\
\text { das } \\
\text { infecções } \\
\text { da corrente } \\
\text { sanguínea }\end{array}$ \\
\hline
\end{tabular}




\begin{tabular}{|c|c|c|c|c|}
\hline & & & relacionadas ao & associada \\
& & & & cateter venoso \\
central (ICSR- & ao cateter \\
venoso \\
& & CVC) em & central \\
& & Unidade de & \\
& & Terapia & \\
& & Intensiva. & \\
\hline
\end{tabular}

Fonte: próprias autoras.

Verificou-se que dos 9 artigos selecionados 4 foram publicados na revista Esc Enfermagem USP, 3 foram publicados na Revista de Enfermagem UFPE, I artigo foi publicado na Revista Texto-Contexto de Enfermagem e I artigo foi publicado na Revista Enferm. Foco.

Em relação à distribuição temporal, percebeu-se que os artigos foram publicados no período de 2017 a 2021, sendo 2 artigos em 2020, I em 2019, 2 em 2018 e 4 artigos em 2017.

Os estudos selecionados foram analisados e agrupados em três áreas temáticas: "Conhecimento dos métodos de prevenção das infecções da corrente sanguínea associada ao cateter venoso central” (2); “Adesão dos profissionais frente aos métodos de prevenção das infecções da corrente sanguínea associada ao cateter venoso central" (4) e "Intervenções para prevenção das infecções da corrente sanguínea associada ao cateter venoso central" (3).

\section{CONHECIMENTO DOS MÉTODOS DE PREVENÇÃO DAS INFECÇÕES DA CORRENTE SANGUÍNEA ASSOCIADA AO CATETER VENOSO CENTRAL}

Entre os estudos que abordaram sobre o conhecimento dos métodos de prevenção das infecções da corrente sanguínea associada ao cateter venoso central podemos citar um estudo transversal, descritivo, com abordagem quantitativa, realizado em três Unidades de Terapia Intensiva. Os dados foram coletados por meio de questionário aplicado face-a-face com 292 profissionais de saúde, tendo como objetivo avaliar conhecimento e comportamento dos profissionais de Unidades de Terapia Intensiva quanto às ações recomendadas no bundle de prevenção de infecção de corrente sanguínea relacionada ao cateter venoso central. Quanto ao conhecimento, o item higienização das mãos apresentou maior nível tanto no momento da inserção como na manutenção. $O$ uso do degermante clorexidina, seguido por alcoólico e datarhub ou conectores foram os itens de menor conhecimento. Quanto ao comportamento, os profissionais relataram: sempre usar a 
paramentação correta para inserção do cateter $(84,25 \%)$, nunca esperar a secagem do antisséptico antes de inserir o cateter $(25,34 \%)$ e nunca realizar limpeza do hub ou conectores com álcool $70 \%(23,86 \%)$. Os resultados demonstraram que o conhecimento e comportamento dos profissionais em relação ao bundle de cateter venoso central apresentaram fragilidades, revelando a importância de incentivar programas de capacitação nesta área do conhecimento (COSTA et al, 2020).

Outro artigo que abordou esse assunto, foi um estudo quantitativo de corte transversal que teve como objetivo avaliar o conhecimento autorreferido das equipes médica e de enfermagem quanto às medidas de prevenção de infecção da corrente sanguínea relacionada a cateter venoso central. Tratou-se de um estudo realizado na unidade de terapia intensiva de um hospital público de urgência e emergência de Belo Horizonte, Minas Gerais. A população foi composta por médicos, enfermeiros e técnicos de enfermagem responsáveis pela inserção e manutenção dos cateteres venosos centrais. Em relação aos resultados, verificou-se que quanto aos treinamentos sobre a infecção da corrente sanguínea relacionada ao CVC, 93 profissionais (71\%) informaram não terem recebido treinamento no ano de 2015 e 12 (9,2\%) referiram não lembrar (SILVA; OLIVEIRA, 2018).

Considerando as medidas autorreportadas para prevenir a infecção da corrente sanguínea antes da inserção do CVC pela equipe médica, todos os entrevistados afirmaram utilizar medidas de barreira como: luvas estéreis, máscara, gorro, capote e campo estéril, preparo cirúrgico das mãos e antissepsia da pele do paciente. $\mathrm{O}$ antisséptico mais referido para a antissepsia da pele foi a clorexidina degermante seguida da alcoólica e o sítio anatômico indicado para inserção do CVC foi a veia subclávia (50\%) e jugular (50\%). Quanto ao critério utilizado para remoção do CVC inserido em situação de urgência, a maioria (52,6\%) informou que a substituição do cateter deve ocorrer o mais rápido possível. As demais respostas foram: se o paciente apresentar febre (21\%) e que não há critérios para substituição (26,4\%) (SILVA; OLIVEIRA, 2018).

As principais medidas relatadas pela equipe de enfermagem para prevenir a infecção da corrente sanguínea, durante a manipulação do CVC, foram uso de luvas (55,1\%), utilização de antisséptico ao trocar o curativo (51,4\%), higienização das mãos (36,7\%), curativo estéril $(29,4 \%)$ e troca do curativo conforme protocolo da unidade $(28,4 \%)$. No 
que se refere à desinfecção do hub antes de administrar medicamentos pelo CVC, essa prática foi relatada por $55 \%$ da equipe. Em contrapartida, 53,3\% informaram que não há tempo determinado para realizar a desinfecção. $\mathrm{Na}$ troca do curativo foram mencionadas a utilização de luvas estéreis $(79,8 \%)$ e a avaliação diária do sítio de inserção do CVC pela maioria dos profissionais (96,3\%) (SILVA; OLIVEIRA, 2018).

Ao questionar as equipes médica e de enfermagem quanto ao curativo do sítio de inserção do CVC, as coberturas citadas foram gaze estéril e filme transparente (89,3\%) e o antisséptico mais informado foi a associação da clorexidina degermante seguida da alcoólica (75,6\%). Relativo ao conceito de bundle, apenas 24 profissionais (18,3\%) afirmaram conhecer e, desses, I3 conseguiram conceituar o termo (SILVA; OLIVEIRA, 2018).

Logo, na análise global das questões, constatou-se um conhecimento limitado às medidas consideradas padrão ouro na prevenção de infecção da corrente sanguínea relacionada ao cateter venoso central, reforçando a importância de mais investimentos na discussão da prevenção dessa infecção, bem como na educação permanente (SILVA; OLIVEIRA, 2018).

\section{ADESÃO DOS PROFISSIONAIS FRENTE AOS MÉTODOS DE PREVENÇÃO DAS INFECÇÕES DA CORRENTE SANGUÍNEA ASSOCIADA AO CATETER VENOSO CENTRAL}

Em relação aos estudos que abordaram a adesão dos profissionais frente aos métodos de prevenção das infecções da corrente sanguínea associada ao cateter venoso central, destacou-se um estudo quantitativa, transversal, observacional e descritivo, desenvolvido em um hospital de ensino de porte especial, em seis unidades de Terapia Intensiva, que teve como objetivo verificar a adesão da equipe de enfermagem ao protocolo de infecção de corrente sanguínea em pacientes em uso do CVC. Concluiu-se, nesse estudo, uma alta adesão da equipe de enfermagem ao protocolo de prevenção da corrente sanguínea, em relação ao alto índice de conformidade das ações preventivas, como identificação, condições de higiene e fixação do CVC, identificação do equipo e presença de fita colorida no CVC. Constatou-se, também, baixo índice de infecção de corrente sanguínea relacionado ao CVC comparado a literatura (CRIVELARO et al, 2018).

Também foi analisado um estudo quase-experimental, realizado na Unidade de Terapia Intensiva de um hospital de urgência e emergência, que teve como objetivo 
verificar a adesão da equipe multiprofissional para as medidas de prevenção da infecção da corrente sanguínea relacionada ao cateter venoso central. Foi possível concluir, nesse estudo, que durante a observação direta dos profissionais, a higiene das mãos antes e após a realização dos procedimentos e a desinfecção do hub antes de administrar medicamentos, não foram aderidas completamente pela equipe de enfermeiros e técnicos de enfermagem, porém, em relação a equipe médica, observou-se maior adesão ao utilizar todas as medidas de barreira que são preconizadas durante a inserção do CVC, tanto no período pré intervenção, quanto no pós intervenção. Diante dos dados apresentados, salientou-se a importância de maior investimento na discussão da prevenção da infecção da corrente sanguínea, bem como na educação continuada das equipes em suas fragilidades, especialmente, em relação às medidas de manutenção do CVC (SILVA; OLIVEIRA, 2017).

Outro estudo foi realizado em duas Unidades de Terapia Intensiva (uma neonatal com 20 leitos e outra pediátrica com ıo leitos) de um hospital de grande porte de Belo Horizonte. A população foi constituída de 188 neonatos e crianças internadas entre fevereiro e setembro de 20r6, que foram submetidos a instalação do CVC. O tamanho amostral foi de 54 pacientes e cinco deles foram submetidos a mais de um procedimento, totalizando 59 observações. Tratou-se de um estudo descritivo exploratório, de abordagem quantitativa, que teve como objetivo descrever o comportamento observado dos profissionais da Unidade de Terapia Intensiva Neonatal e Pediátrica, segundo os itens do bundle de inserção de cateter venoso central, bem como o perfil clínico e de nascimento de neonatos e crianças que receberam o CVC (ARAÚJO et al, 2017).

Este estudo mostrou que quase a totalidade dos profissionais de saúde, em algum momento durante os procedimentos observados, não cumpriu etapas importantes do bundle de inserção do CVC. Destacaram-se as técnicas incorretas na realização da antissepsia cirúrgica e no uso de clorexidina degermante e alcoólica. Foi possível concluir que é necessário reforçar a importância de um maior investimento na educação permanente da equipe referente as ações de prevenção de infecção de corrente sanguínea relacionada ao cateter venoso central, para que se reduza a ocorrência de eventos adversos relacionados ao uso da terapia intravenosa (ARAÚJO et al, 2017). 
Por fim, ainda pertinente à esta área temática, foi selecionada para análise, uma pesquisa com delineamento descritivo, exploratório, de abordagem quantitativa, desenvolvido em um hospital escola, situado no município de Campina Grande (PB), no período de janeiro a fevereiro de 2017. O local de estudo foi uma UTI adulto que dispunha de io leitos, sendo um destinado a pacientes que necessitassem de isolamento e a população foi composta por 30 profissionais da equipe de enfermagem. Utilizou-se um cheklist para a observação sistemática construída conforme as diretrizes nacionais da ANVISA, Guideline do Centers for Disease Control and Prevention e Bundle do Institute for Healthcare Improvimient. O objetivo do estudo foi avaliar o conhecimento e adesão da equipe de enfermagem às medidas de prevenção de infecções de corrente sanguínea relacionadas ao cateter venoso central (ICRS-CVC) em UTI adulto (DANTAS et al, 2017).

Pode-se concluir, nesse estudo, que a equipe de enfermagem possui fragilidades no conhecimento das medidas de prevenção de ICRS-CVC que são preconizadas pela ANVISA e pelos órgãos internacionais. Percebeu-se também que grande parte dos profissionais, assinalou não ter tido nenhum treinamento voltado às medidas de prevenção da ICRS-CVC. A maioria dos profissionais de enfermagem relatou realizar medidas de manutenção do CVC, como desinfecção de ampolas, frasco ampolas e do hub do cateter antes de administrarem os medicamentos, porém, não foram visualizados tais procedimentos durante o período de observação aos cuidados prestados pela equipe. Os profissionais também demonstraram fragilidade na adesão às medidas de prevenção da ICRS-CVC, relataram dúvidas no manejo do CVC e desconhecimento sobre as medidas de prevenção da ICRS, reiterando, assim, a necessidade dos gestores implementarem medidas de educação continuada para esses profissionais sobre o assunto (DANTAS et al, 2017).

\section{INTERVENÇÕES PARA PREVENÇÃO DAS INFECÇÕES DA CORRENTE SANGUIINEA ASSOCIADA AO CATETER VENOSO CENTRAL}

Apenas três artigos abordaram as intervenções para prevenção das infecções da corrente sanguínea associada ao cateter venoso central.

Um deles teve como objetivo verificar as medidas utilizadas em Unidades de Terapia Intensiva para prevenção de infecção relacionada à assistência à saúde. Assim, 
constatou-se que dentre as categorias temáticas estabelecidas para as medidas de prevenção de infecção da corrente sanguínea, os cuidados como preparo da pele, inserção, higienização das mãos, medidas educativas e uso de cobertura foram as mais frequentes mencionadas (DANTAS et al, 2020).

O outro artigo analisado apresentou delineamento quantitativo, de corte transversal, em um hospital público, com a participação de 82 profissionais da saúde da equipe intensivista. $\mathrm{O}$ objetivo foi verificar o conhecimento dos profissionais intensivistas sobre o bundle para prevenção de Infecção da Corrente Sanguínea relacionado ao Cateter Venoso Central e sobre os cuidados no manejo deste dispositivo. Diante do que foi estudado adverte-se que embora a maior parte dos entrevistados tenha mostrado um conhecimento satisfatório em relação ao bundle e aos cuidados no manejo dos cateteres, um percentual significativo demonstrou fragilidades ainda existentes (FERNANDES et al, 2019).

Já, o terceiro e último estudo sobre essa temática, tratou-se de um estudo de intervenção realizado na Unidade de Terapia Intensiva de um hospital universitário, com os membros das equipes de enfermagem e médica, de junho a dezembro de 2014. Foram aplicados os quatro passos da metodologia Positive Deviance: Definir, Determinar, Descobrir e Desenhar. O objetivo foi descrever a aplicação do Positive Deviance como estratégia na prevenção e no controle da infecção de corrente sanguínea. Pode-se concluir que o Positive Deviance auxiliou na implementação de propostas de melhorias de processo de trabalho e no desenvolvimento da equipe para os problemas identificados no cuidado com o cateter venoso central (OLIVEIRA et al, 2017).

\section{CONCLUSÃO}

Os resultados mostraram que dos 9 artigos selecionados, nesta revisão de literatura, 4 abordaram sobre a adesão dos profissionais frente aos métodos de prevenção das infecções da corrente sanguínea associada ao cateter venoso central; 3 estavam relacionados às intervenções para prevenção das infecções da corrente sanguínea associada ao cateter venoso central e 2 ao conhecimento dos métodos de prevenção das infecções da corrente sanguínea associada ao cateter venoso central. 
Levando-se em consideração o conhecimento, constatou-se que os profissionais apresentaram fragilidades quanto às medidas consideradas padrão ouro na prevenção de infecção da corrente sanguínea relacionada ao cateter venoso central, reforçando a necessidade de investimento em capacitação.

Apesar das divergências apresentadas pelos estudos relacionados à adesão dos profissionais, frente aos métodos de prevenção das infecções da corrente sanguínea, verificou-se que a maioria registrou que as medidas de prevenção não são cumpridas em todas as suas etapas, que existem dúvidas, falta de conhecimento e de treinamento referente a esta temática.

Quanto às intervenções para prevenção das infecções da corrente sanguínea associada ao cateter venoso central, observou-se que, apesar de a maioria dos profissionais, participantes das pesquisas, afirmar ter conhecimento sobre as intervenções necessárias, foi identificado que nem sempre essas medidas são adotadas na prática.

Conclui-se, portanto, que se torna importante a implantação de ações de educação permanente acerca das medidas de prevenção de infecção de corrente sanguínea associada ao uso de cateter venoso central.

\section{REFERÊNCIAS}

ANVISA - Agência Nacional de Vigilância Sanitária. Corrente Sanguínea: Critérios Nacionais de Infecções Relacionadas à Assistência à Saúde. Unidade de Investigação e Prevenção das Infecções e dos Efeitos Adversos - UIPEA Gerência Geral de Tecnologia em Serviços de Saúde - GGTES. Setembro, 2009. Disponível em: https://www.anvisa.gov.br/servicosaude/manuais/correntesanguinea.pdf. Acesso em: $02 / 03 / 2021$.

ANVISA - Agência Nacional de Vigilância Sanitária. Critérios Diagnósticos de Infecção Relacionada à Assistência à Saúde. In: Série Segurança do Paciente e Qualidade em Serviços de Saúde [Internet]. 2017 p.I86. Disponível em: http://portal.anvisa.gov.br/documents/33852/3507912/Caderno+2++Critérios+Diagnósticos+de+Infecção+Relacionada+à+Assistênciaà+Saúde/7485b45a-o74f4b34-8868-6ifies724501. Acesso em: 02/03/2021. 
ARAÚJO, F.L.; MANZO, B.F.; COSTA, A.C.L.; CORRÊA, A.R.; MARCATTO, J.O.; SIMÃO, D.A.S Adesão ao bundle de inserção de cateter venoso central em unidades neonatais e pediátricas. Rev Esc Enferm USP.51:e03269, 2017. Disponível em: http://www.revenf.bvs.br/scielo.php?script=sci_arttext\&pid=Soo8o-62342017000100453. Acesso em: 26/03/2021.

CATARINO, C.F.; MARTINS, A.C.S.; SILVA, A.P.A.M.; GOMES, A.V.O.; NASCIMENTO, M.A.L. Perfil epidemiológico das infecções primárias de corrente sanguínea em uma unidade de Terapia Intensiva Neonatal. R. pesq.: cuid. fundam.jan/mar.5(I):3229-37, 2012. Disponível em: http://www.seer.unirio.br/index.php/cuidadofundamental/article/view/2013/pdf_679. Acesso em: 02/03/2021.

CDC - Center for Disease and Prevention Control. NationalHealthcare Safety Network (NHSN) Overview Patient Safety Component Manual, 20r6. Disponível em: www.cdc.gov/nhsn/PDFs/HPS-manual/HPS_Manual-exp-plus-flu-portfolio.pdf. Acesso em: 02/03/2021.

COSTA, C.A.B.; ARAÚJO, F.L.; COSTA, A.C.L.; CORRÊA, A.R.; KUSAHARA, D.M.; MANZO, B.F. Bundle de Cateter Venoso Central: conhecimento e comportamento de profissionais em Unidades de Terapia Intensiva adulto. Rev Esc Enferm USP.54e03629, 2020. Disponível em: https://www.scielo.br/scielo.php?script=sci_arttext\&pid=Soo8o62342020000100472. Acesso em: 26/03/2021.

CRIVELARO， N.; CONTRIN, L.M.; BECCARIA， L.M.; FRUTUOSO, I.S.; SILVEIRA, A.M.; WERNECK, A.L. Adesão da enfermagem ao protocolo de infecção de corrente sanguínea. Rev enferm UFPE. Recife, I2(9):2361-7, 2018. Disponível em: https://periodicos.ufpe.br/revistas/revistaenfermagem/article/view/234886/29919.

Acesso em: 26/03/2021.

DANTAS, A.C.; ARAÚJO, J.N.M.; NASCIMENTO, A.A.A.; MARTINS, Q.C.S.; ARAÚJO, M.G. Measures used in intensive care units to prevent infection: an integrative 
review. Rev Rene.21:e44043, 2020. Disponível em: http://periodicos.ufc.br/rene/article/view/44043/16i812. Acesso em: 26/03/2021.

DANTAS, G.D.; FIGUEIREDO, D.S.T.O.; NOBRE, A.M.D.; PIMENTEL, E.R.S. Adesão da equipe de enfermagem às medidas de prevenção de infecções de corrente sanguínea. Rev enferm UFPE. Recife,II(I0):3698-706, 2017. Disponível em: https://periodicos.ufpe.br/revistas/revistaenfermagem/article/view/15018/24286. Acesso em: 26/03/2021.

ERCOLE, F.F.; MELO, L.S.; ALCOFORATO, C.L.G.C. Revisão integrativa versus revisão sistemática. Reme. Rev Min Enferm.18(I):I-260, 2014.

FERNANDES, A.T.; RIBEIRO, N.F. Infecção do Acesso Vascular. In: Fernandes AT, Fernandes MA, Ribeiro N F, organizadores. Infecção Hospitalar e suas Interfaces na Área da Saúde. São Paulo (SP): Atheneu; p. 556-79, 2000.

FERNANDES, M.S.; FERNANDES, M.S.; NOGUEIRA, H.K.L.; PONTES, F.S.; GÓES, A.C.F. OLIVEIRA, D.F. Bundle para a prevenção de infecção de corrente sanguínea. Rev enferm UFPE. Recife,I3(I):I-8, 2019. Disponível em: https://pesquisa.bvsalud.org/portal/resource/pt/biblio-Ioo5889. Acesso em: 26/o3/202I.

MARQUES NETTO, S.; ECHER, I.C.; KUPLICH, N.M.; KUCHENBECKER, R.S.; KESSLER, F. Infecção de cateter vascular central em pacientes adultos de um centro de terapia intensiva. Rev Gaúcha Enferm., Porto Alegre (RS), set;30(3):429-36, 2009. Disponível em: https://www.lume.ufrgs.br/bitstream/handle/IoI83/23638/ooo731557.pdf?sequence =I\&isAl lowed=y. Acesso em: 02/03/2021.

MENDONÇA, K.M.; NEVES, H.C.C.; BARBOSA, D.F.S.; SOUZA, A.C.S.; TIPPLE, A.F.V.; PRADO, M.A. Atuação da enfermagem na prevenção e controle de infecção de corrente sanguínea relacionada a cateter. Revista de enfermagem UERJ, Rio de Janeiro; 
I9(2):330-333,

2011.

Disponível

em:

https://repositorio.bc.ufg.br/bitstream/ri/r6244/5/Artigo\%20-

\%20Katiane\%2oMartins\%2oMendon\%C3\%A7a\%20-\%20201r.pdf. Acesso em: 02/03/2021.

OLIVEIRA, F.T.; FERREIRA, M.M.F.; ARAÚJO, S.T.C.; BESSA, A.T.T.; MORAES, A.C.B.; STIPP, M.A.C. Positive deviance as a strategy to prevent and control bloodstream infections in intensive care. Rev Esc Enferm USP. 51:e03212, 2017. Disponível em: https://www.scielo.br/pdf/reeusp/v51/pt_1980-220X-reeusp-51-e03212.pdf. Acesso em: 26/03/2021.

PIEROTTO, A.A.S. Infecção de corrente sanguínea relacionada ao uso de cateteres venosos centrais em pacientes pediátricos de um hospital terciário. (Dissertação de Mestrado). Porto Alegre: Pontifícia Universidade Católica do Rio Grande do Sul; 2015. ROSADO, V.; ROMANELLI, R.M.; CAMARGOS, P.A. Risk factors and preventive measures for catheter-related bloodstream infections. J Pediatr (Rio J). Nov-Dez; 87 (6): 469-77, 2011. Disponível em: http://www.jped.com.br/ArtigoDetalhe. aspx?varArtigo=2264\&idioma=pt-BR. 02/03/202I.

SANTOS, E.R.; LEAL, R.; CAVALHEIRO, A.M. Cateter venoso central. Knobel E. Terapia Intensiva: Enfermagem. São Paulo: Atheneu; p.I89-196, 2006.

SILVA, A.G.; OLIVEIRA, A.C. Adesão às medidas para prevenção da infecção da corrente sanguínea relacionada ao cateter venoso central. Enferm. Foco.8 (2): 36-4I, 2017. Disponível em: http://biblioteca.cofen.gov.br/wpcontent/uploads/2017/o7/Ades\%C3\%A30-\%C3\%Aos-medidas-parapreven\% $\mathrm{C}_{3} \% \mathrm{~A}_{7 \%} \mathrm{C}_{3} \% \mathrm{~A}_{30}$-da-infec\% $\mathrm{C}_{3} \% \mathrm{~A}_{7 \%} \mathrm{C}_{3} \% \mathrm{~A}_{30}$-da-corrente-sangu\% $\mathrm{C}_{3} \% \mathrm{AD}$-arelacionada-ao-cateter-venoso-central.pdf. Acesso em: 26/03/2021.

SILVA, A.G.; OLIVEIRA, A.C. Conhecimento autorreferido das equipes médica e de enfermagem quanto às medidas de prevenção de infecção da corrente sanguínea. Texto Contexto Enferm. 27(3):e3480017, 2018. Disponível em: https://www.scielo.br/scielo.php?pid=So104-07072018000300327\&script=sci_arttext. Acesso em: 26/03/2021. 
SILVEIRA, R.C.C.P.; BRAGA, F.T.M.M.; GARBIN, L.M.; GALVÃO, C.M. O uso do filme transparente de poliuretano no cateter venoso central de longa permanência. Rev. Latino-Am. Enfermagem nov-dez;18(6): [09 telas], 2010. Disponível em: https://www.scielo.br/j/rlae/a/nvxTLXgfnxQMm3whC6JDYtd/?format=pdf\&lang=pt\# : :text=O\%2ocurativo\%2ode\%2opoliuretano\%2ofoi,em\%2otr\%C3\%AAs\%2ocasos\%20com\% 2ofilme. Acesso em: 02/03/2021 